\title{
Heterodyne laser Doppler vibrometers integrated on silicon-on-insulator based on serrodyne thermo-optic frequency shifters
}

\author{
Yanlu Li, ${ }^{*}$ Steven Verstuyft, Gunay Yurtsever, Shahram Keyvaninia, \\ Gunther Roelkens, Dries Van Thourhout, and Roel Baets \\ Photonics Research Group, Department of Information Technology (INTEC), Ghent-University—Interuniversitair \\ Micro-Elektronica Centrum (IMEC), \\ Sint-Pietersnieuwstraat 41, Ghent 9000, Belgium
}

Center for Nano- and Biophotonics (NB-Photonics), Ghent University, Sint-Pietersnieuwstraat 41, Ghent 9000, Belgium

*Corresponding author: Yanlu.Li@intec.ugent.be

Received 20 June 2012; revised 2 November 2012; accepted 28 January 2013;

posted 20 February 2013 (Doc. ID 170989); published 1 April 2013

\begin{abstract}
Miniaturized laser Doppler vibrometers (LDVs) have many advantages over conventional bulk LDVs. In this paper, the realization of a miniaturized heterodyne LDV integrated on silicon-on-insulator substrate is reported. The optical frequency shifters in these on-chip LDVs employ a serrodyne technique, and they generate a frequency shift at $2 \mathrm{kHz}$. Vibrations of a mirror for the frequency range between 1.1 and $123 \mathrm{~Hz}$ and the velocity range between 0.8 and $400 \mu \mathrm{m} / \mathrm{s}$ are measured by both an on-chip LDV and a commercial LDV. The measurement results agree well. A compensation method for the influence of on-chip spurious reflections is also demonstrated. () 2013 Optical Society of America

OCIS codes: $120.7250,130.0130$
\end{abstract}

\section{Introduction}

Laser Doppler vibrometry (LDV) is a technique used for measuring the velocity of a moving surface, and it is basically the same technique as laser Doppler velocimetry. The term "vibrometry" is used to emphasize that this technique is mainly used to measure vibrations of a surface. Thanks to the ability to conduct noncontact vibration measurements, LDV has drawn much attention in various industrial applications and research fields. In order to reduce the fabrication cost, increase the mobility, and improve the performance, many efforts have been made to miniaturize LDV systems $[1,2]$. The miniaturization also makes it easier to realize multipoint LDV systems, which can measure vibrations on several different positions simultaneously (e.g., dual-beam LDV used for measuring pulse wave velocity in elastic vessels

$1559-128 X / 13 / 102145-08 \$ 15.00 / 0$

(C) 2013 Optical Society of America
[3]). We propose to use the silicon-on-insulator (SOI) photonic integrated circuit (PIC) technology for the miniaturization of LDV systems. The SOI technology makes it possible to dramatically reduce the footprint of the PICs [4]. An LDV PIC on SOI with a footprint of less than $0.4 \mathrm{~mm}^{2}$ is demonstrated in this paper. The core of an LDV PIC is an optical interferometer. Because this interferometer is implemented in a chip, its arm lengths are not sensitive to environmental vibrations. Environmental temperature variations also have a weak influence on the performance of this interferometer because the arms are designed to be short and close enough. These benefits make an LDV PIC more stable compared to a free-space or fiber-based LDV [5]. In case of high volume production, the cost of miniaturized LDVs on SOI can also be considerably lower than that of more conventional implementations.

In our proposed design, a waveguide-based Michelson interferometer is fabricated on an SOI chip. An on-chip grating coupler is placed in one arm of the 
interferometer so as to couple light out of the chip and collect the portion of light that is reflected by a vibrating surface. To the best of our knowledge, this kind of integrated LDV has not yet been reported before. Because the grating coupler and optical splitters/combiners in the interferometer introduce spurious reflections, which may deteriorate the output of the LDV, it is interesting to understand the impact of these spurious reflections and develop a method to compensate for the corresponding deviations. The on-chip LDV PIC reported in this paper employs an optical heterodyne detection approach [6] in which an optical frequency shifter (OFS) is used in the reference arm to generate a constant frequency shift $f_{\text {ofs }}$. The frequency shift helps to discriminate the movement direction of the target and circumvents low-frequency noise. The acousto-optic effect is a common method used for generating the frequency shift in free-space and fiber-based LDV systems [5]. However, because the piezoelectric effect is absent in unstrained crystalline silicon, it is difficult to generate an acoustic wave on SOI. To avoid this problem, we propose to use serrodyne modulation $[7,8]$ for creating a frequency shift. Carrier injection/ depletion-based phase modulators [9] have a speed that is high enough to generate different serrodyne frequency shifts for commercial use. However, an additional intensity modulation coexists with the phase modulation in this kind of modulators, which cannot be ignored in the serrodyne OFS. In our proof-ofprinciple design, the serrodyne modulation is implemented by means of an integrated thermo-optic (TO) phase modulator, which introduces a negligible intensity modulation. In this case, the influence of imperfect phase modulations can be excluded.

The TO phase modulator reported in this paper is similar to the one reported in [8]. It comprises a deeply etched optical waveguide $(220 \mathrm{~nm}$ high and $450 \mathrm{~nm}$ wide) and a titanium heater (750 $\mu \mathrm{m}$ long and $2.25 \mu \mathrm{m}$ wide). A benzocyclobutene (BCB) layer with a thickness of around $400 \mathrm{~nm}$ is inserted between the waveguide and the heater to avoid strong absorption of the guided light in the waveguide by the metal (see Fig. 1). However, measurement results shown that the guided light still had a $3 \mathrm{~dB}$ power loss, which was caused by the absorption of the $750 \mu \mathrm{m}$ long heater. A repetitive square-root-of-time $(\mathrm{SQOT})$ voltage $V(t)=V_{\mathrm{pp}} \sqrt{t f_{\mathrm{ofs}}-\text { floor }\left(t f_{\mathrm{ofs}}\right)}$ was

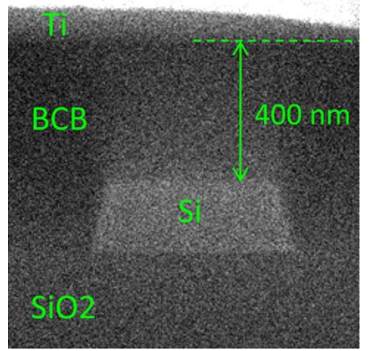

Fig. 1. (Color online) Scanning electron microscopy image of the heater cross section.
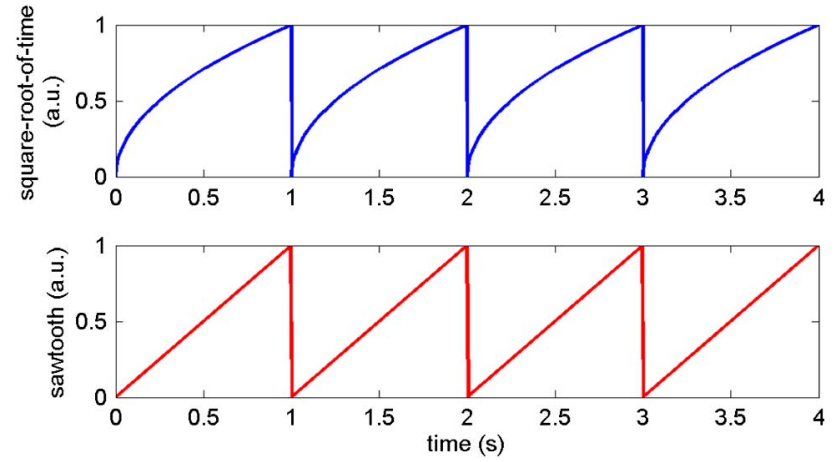

Fig. 2. (Color online) Example of the square-root-of-time (SQOT) and sawtooth signals with a frequency at $1 \mathrm{~Hz}$. When the voltage signal applied across the heater has a SQOT profile, the phase profile of the optical signal should be a sawtooth.

applied across the heater (the voltage shape is plotted in Fig. 2), where floor $(x)$ is a floor function that returns the largest integer not greater than $x$. A temperature modulation was thus created in the underlying waveguide, and a sawtooth profile was generated in the phase of the guided light. This modulation leads, under perfect conditions, to a pure phase shift. However, under an imperfect modulation, the modulated optical signal can be expressed as

$$
E_{\mathrm{ofs}}(t)=\sum_{k} \mu_{k} \exp \left[i 2 \pi\left(f_{0}+k \cdot f_{\mathrm{ofs}}\right) t\right],
$$

where $k$ is the order of the harmonic. For a pure frequency shift, all the harmonics with $k \neq 1$ should be strongly suppressed. However, in practice, only the harmonics with $k$ equal to -1 and 0 need be suppressed because the other nonfundamental orders can normally be filtered away in a later stage. The simulated power spectral densities of several different harmonic orders as a function of the peak-to-peak phase amplitude $\theta_{\mathrm{pp}}$ are plotted in Fig. $\underline{3}$. It is found that when $\theta_{\mathrm{pp}}$ is near $2 \pi$, the power of the fundamental harmonic is close to the maximal value while the other harmonic orders are strongly suppressed. It can also be found that the power will be redistributed from the fundamental harmonic into the secondorder harmonic as $\theta_{\mathrm{pp}}$ is shifting from $2 \pi$ to $4 \pi$

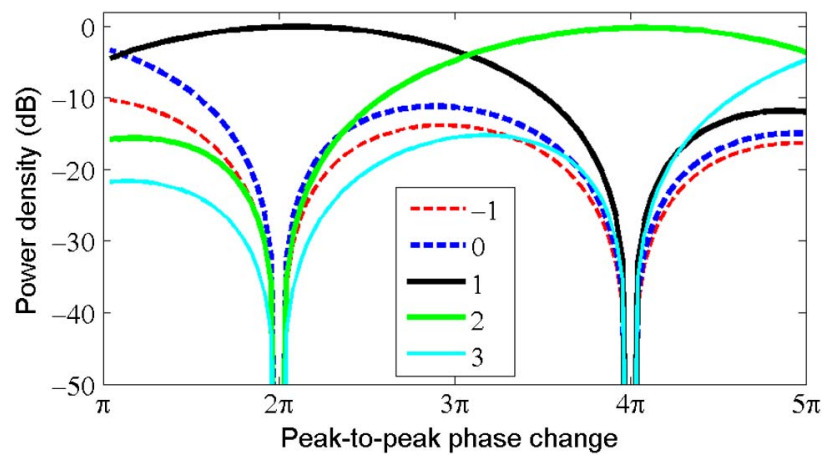

Fig. 3. (Color online) Different harmonic orders as a function of the peak-to-peak phase change (simulation results). 


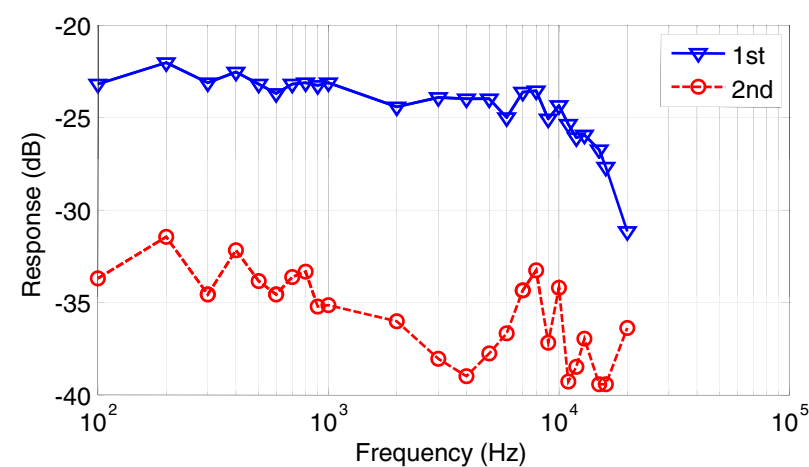

Fig. 4. (Color online) Measured relations between the power of different harmonics (fundamental and the second order) and the value of the frequency shift when a serrodyne technique is applied to our TO phase modulator.

because the phase slope in the sawtooth ramp is doubled. According to these results, in order to generate a frequency shift with most of the power concentrated on the fundamental harmonic, the peak-to-peak voltage $V_{\text {pp }}$ should be adjusted so that the sawtooth amplitude of the optical phase $\theta_{\mathrm{pp}}$ becomes $2 n \pi, n \in N$. For the fabricated device, the value of $V_{\mathrm{pp}}$ applied to the phase modulator was $3.75 \mathrm{~V}$. Because the resistance of the heater was around $1 \mathrm{k} \Omega$, the average power consumption of the heater was around $7 \mathrm{~mW}$. The performance of the serrodyne frequency shift is also limited by the speed of the phase modulator, which has been explained in [8]. The influence of the slow TO phase modulator used in our design on a serrodyne modulation is illustrated in Fig. $\underline{4}$, which shows the measured relations between the power of different harmonics (fundamental and the second order) and the value of the frequency shift when a serrodyne technique is applied to our TO phase modulator. It can be seen that the $3 \mathrm{~dB}$ cutoff frequency of the fundamental harmonic is below $10 \mathrm{kHz}$. In this paper, the frequency shift $f_{\text {ofs }}$ is kept at $2 \mathrm{kHz}$.

Compared to our early work reported in [7], where we demonstrated a fiber-based implementation based on the same concept, this paper focuses on SOI-implementation and the devices on the SOIchip. The spurious reflections of these devices (e.g., optical splitters, grating couplers) are relatively strong, and they can cause an apparent influence in the LDV output. Besides, the coupling efficiency of the simple grating coupler used in this work is not very high (around 30\%-40\%), which may introduce a too high suppression in the useful reflected light and deteriorate the LDV output. These problems are analyzed in this paper. The PIC design and the measurement setup will be described in Section 2. In Section $\underline{3}$, the measurement results of the on-chip LDV will be compared with a commercial LDV.

\section{Design and Measurement Setup}

An external distributed feedback (DFB) laser is used in our design instead of an integrated version,

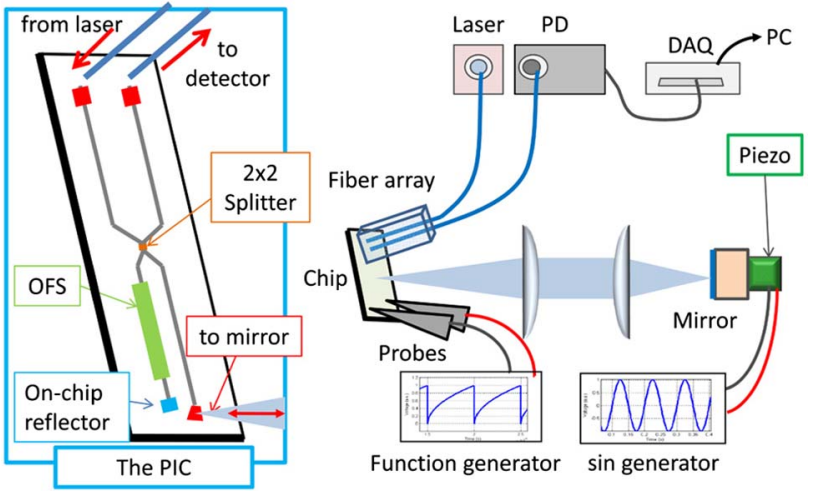

Fig. 5. (Color online) PIC design and the setup used to measure mirror vibrations. A Michelson-type LDV PIC is shown in the box on the left side. PD, photodetector; PC, personal computer; and DAQ, data acquisition card.

although integration of DFB lasers on an SOIplatform is possible and has been demonstrated [10]. The PIC design of the Michelson-type LDV and the measurement setup are schematically shown in Fig. 5 . In this setup, coherent light with a wavelength of $1550 \mathrm{~nm}$ and a $3 \mathrm{~dB}$ linewidth of about $2 \mathrm{MHz}$ is sent into the on-chip interferometer via a standard on-chip grating coupler. The light is split into two paths by a $2 \times 23 \mathrm{~dB}$ multimode-interference (MMI) coupler. The measurement light is sent out of the chip via a tilted grating coupler, which is designed to suppress on-chip reflections [11]. Microscope images of both the MMI and the tilted focusing grating coupler are shown in Fig. $\underline{6}$. With the help of two aspherical lenses, the measurement light is focused perpendicularly onto a vibrating mirror attached to a piezo stack. The reflected light from the mirror is recaptured by the same tilted grating coupler on the chip. The round-trip distance of the light in free space is about $40 \mathrm{~cm}$, which is much less than the coherence length of the light. Because the grating coupler is polarization sensitive, only the reflected light with the correct polarization can be coupled back to the chip. A serrodyne OFS and an on-chip reflector are put in the reference arm (see Fig. 6). The signals reflected from both the measurement and the reference arms are recombined in the same $2 \times 2 \mathrm{MMI}$ as mentioned before and are sent to an external photodetector via another grating coupler.

The photocurrent of the recombined signal is expressed as

$$
\begin{aligned}
i(t) & \propto\left|\alpha(t) \cdot e^{j\left[2 \pi f_{0} t+\theta(t)\right]}+e^{j 2 \pi\left(f_{0}+f_{\text {ofs }}\right) t}\right|^{2} \\
& =1+\alpha^{2}(t)+2 \alpha(t) \cos \left[2 \pi f_{\text {ofs }} t-\theta(t)\right],
\end{aligned}
$$

where $f_{0}$ is the frequency of the coherent light, $\alpha(t)$ is the amplitude of the reflected measurement signal, and $\theta(t)$ is the instantaneous Doppler phase shift due to the vibration of the surface of interest. With a proper demodulator, $\theta(t)$ can be retrieved from this photocurrent signal. The instantaneous 


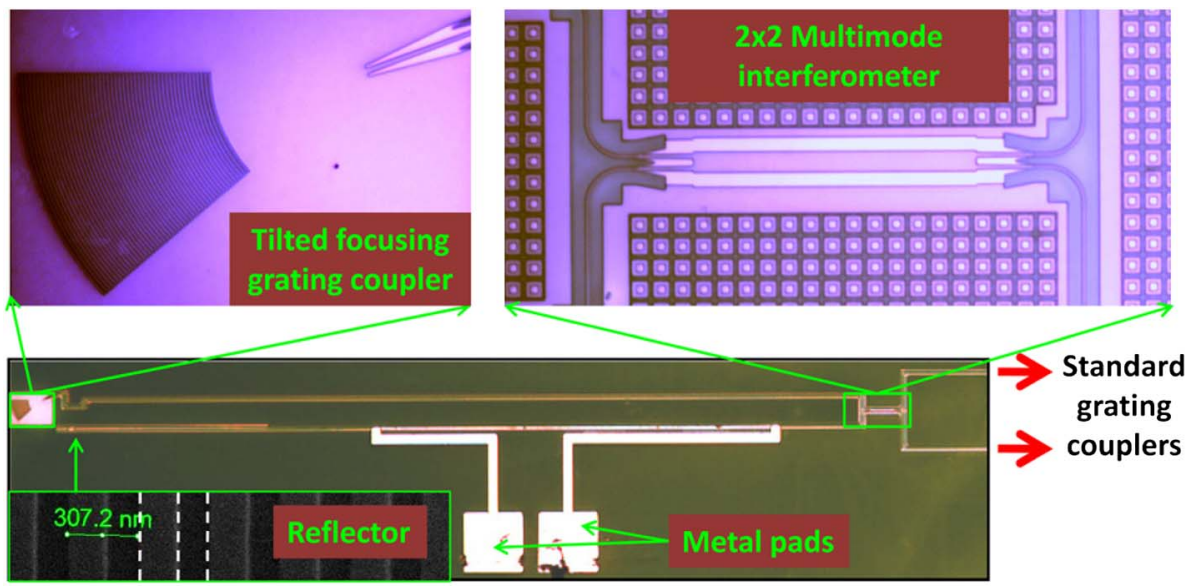

Fig. 6. (Color online) Microscope images of the PIC. Images of the tilted grating coupler [11], $2 \times 2$ MMI coupler, and reflector are also shown.

displacement $d(t)$ of the vibrating surface can be obtained by using the relation $d(t)=\lambda \theta(t) / 4 \pi$, where $\lambda$ is the wavelength of the coherent light in the air.

The in-phase \& quadrature (I\&Q) demodulation is implemented in software. With this method, two signals, $I(t) \propto \alpha(t) \cos [\theta(t)]$ and $Q(t) \propto \alpha(t) \sin [\theta(t)]$, are derived from the photocurrent signal $i(t)$. The angle of the vector $I(t)+i Q(t)$ equals $\theta(t)$. In the measurement results, it is usually found that the trace of the vector is a circle, but the center of the circle is not in the origin, which means that $I(t) \propto \alpha \cos [\theta(t)]+I_{0}$ and $Q(t) \propto \alpha \sin [\theta(t)]+Q_{0}$ [see Fig. 7(a)]. Two main causes are responsible for this deviation: the 0thorder harmonic and spurious reflections. The 0thorder harmonic is normally introduced when the shape of the phase change is not the desired sawtooth profile, and it usually coexists with other harmonic orders. Most of the time the 0th-order harmonic is, however, not strong and can be neglected. The spurious reflection is introduced by the optical components in the interferometer, especially by the on-chip components. For example, a standard grating coupler can introduce a reflection (averaged from 1490 to $1570 \mathrm{~nm}$ ) of around $-20 \mathrm{~dB}$ for light incident from the waveguide onto the grating [12]. The reflection of the commonly used $2 \times 2 \mathrm{MMI}$ is also not negligible. By measuring the ratio between the shift of the I\&Q circle center and the radius of the circle, the relative power strength of the spurious reflection (and the 0th-order harmonic) with respect to the useful reflected light can be estimated. An example of the influence of the $I \& Q$ center shifting caused by the spurious reflection is shown in Fig. 7, and the corresponding root-mean-square (RMS) deviations are plotted as a function of the relative power in the spurious reflection (see Fig. 8). The 0th-order harmonic is not included in the simulation, but it will have the same effect. It can be seen that the influence depends on both the power and the phase of the spurious reflection. A numerical method has been implemented to find the center of the circle $c$ and shift it back to the origin so that the correct phase information can be retrieved. It can be found from Fig. $\underline{8}$ that, by using the compensation method, the

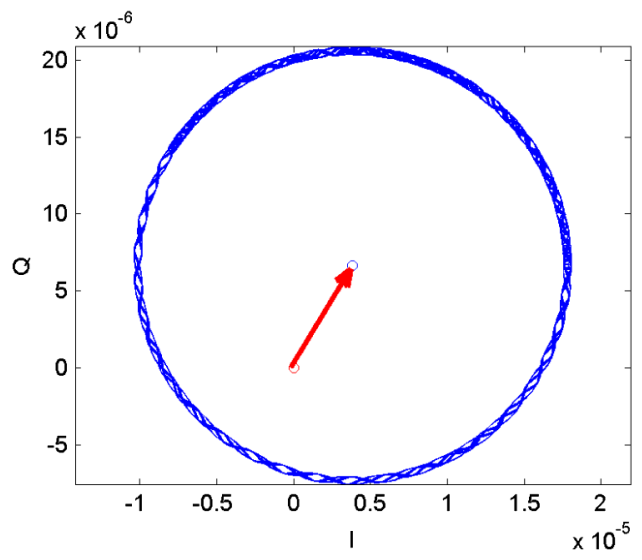

(a)

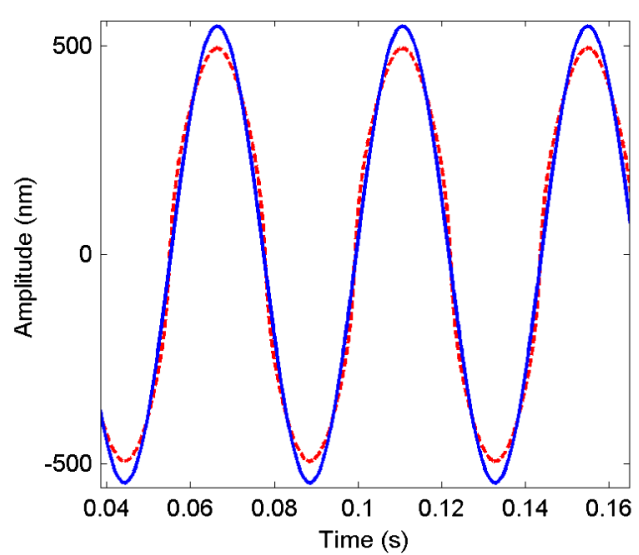

(b)

Fig. 7. (Color online) (a) Influence of the spurious reflection on the demodulated results. In (b), the solid curve represents the original displacement signal and the dashed curve is for the demodulated displacement when a spurious reflection is mixed into the useful reflection signal. In this case the power ratio between the spurious reflection and the useful reflection is $30 \%$, and the vibrating frequency is $22.6 \mathrm{~Hz}$. 


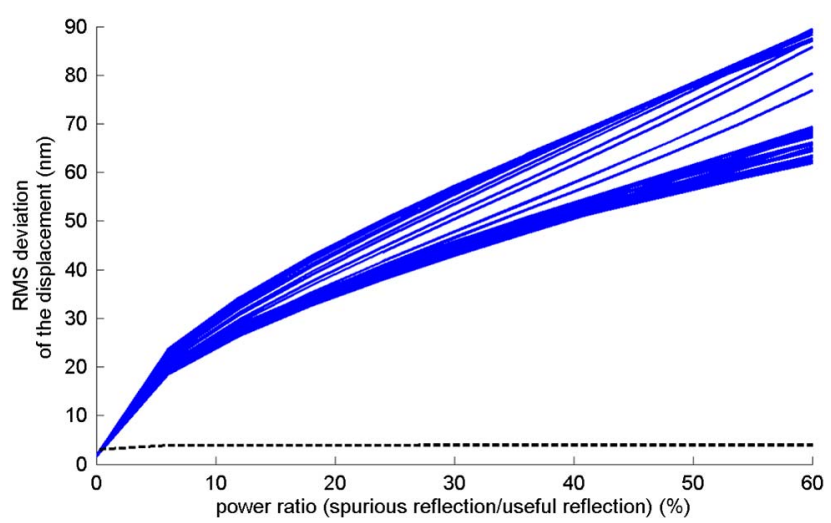

Fig. 8. (Color online) Simulated RMS deviations of the demodulated displacements versus spurious reflections with different power and phase values for the case when the power ratio between the spurious reflection and the useful reflection is $30 \%$. The 36 solid curves represent 36 evenly spaced phases of the spurious reflection from 0 to $2 \pi$. The dashed lines are for the RMS deviation values with the spurious reflection compensation applied.

RMS deviations can be limited within $5 \mathrm{~nm}$. However, this compensation method will not work well when the I\&Q curve is not a nice circle, which can be caused by the amplitude variation in the reflected measurement signal. Due to temperature variations in the waveguide and noise in the laser, the amplitude and phase of the spurious reflection can also change with time, which may make it more difficult to realize a proper compensation. If the driving signal for the OFS is not good enough and the -1storder harmonic is not well suppressed, the I\&Q trace will become an ellipse (see Fig. 9). The eccentricity $e$ of the ellipse is determined by the power ratio between the -1st-order and 1st-order harmonics, with a relation written as

$$
e=2 \sqrt{\mid \mu_{-1} \mu_{1}} /\left(\left|\mu_{1}\right|+\left|\mu_{-1}\right|\right),
$$

where $\mu_{i}(i=-1,1)$ are defined in Eq. 1 . For example, this can happen when the fall time of the sawtooth phase in the frequency-shifted signal is not small enough. The RMS deviations for different fall-time ratios are shown in Fig. 10. When the amplitude of the vibration is smaller than one-half of a wavelength, the $I \& Q$ trace is not a closed circle anymore. In this case, it becomes more difficult to find $c$ and $e$, and the recovery of the vibration values will be difficult or even impossible. The thickness of the edge of the I\&Q curve originates from the varying coupling of the backreflection and noise in the laser.

\section{Measurement Results}

The power spectral density of the optical-frequencyshifted light (combined with a frequency-unshifted light) is plotted in Fig. 11. Results of the recovered displacement measured from the on-chip LDV are compared with those from a commercial LDV [13]. The time-dependent displacements for both measurements are shown in Fig. 12. Note that the two measurements were done separately, and we did not try to match the phases of the results. The curves in Figs. 12(a) and 12(b) are the measurement results of a vibrating piezo stack driven by a sinusoidal voltage signal at $22.6 \mathrm{~Hz}$. The peak-to-peak values of the drive voltages are 50 and $100 \mathrm{~V}$, respectively. In both results, the vibration waves recovered from the onchip LDV are quite similar to those obtained from Polytec, though they are not exactly the same.

The frequency response of this piezo stack was measured by both the on-chip and Polytec LDV systems, and these measurements are shown in Fig. 13. It can be seen that both measured responses are quite similar. However, most of the results from the on-chip LDV were smaller than those from Polytec, and the corresponding ratios were mostly within the region of $90 \%$ and $95 \%$. The cause for this deviation is under further investigation. However, it is possible to correct the on-chip outputs by multiplying the demodulated values with a calibration factor.

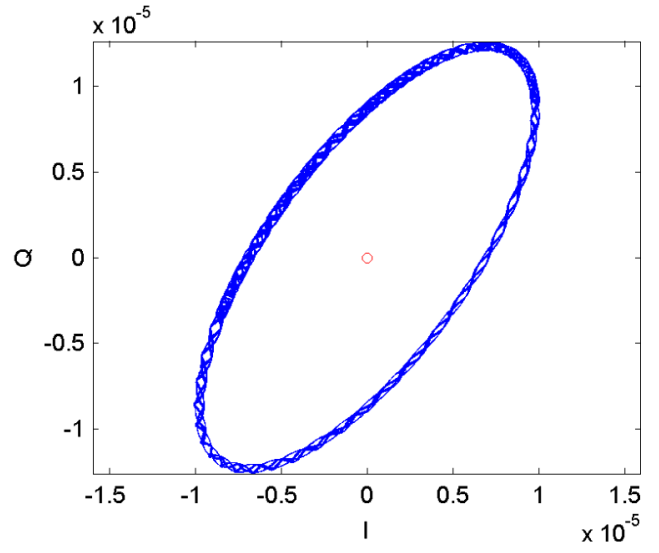

(a)

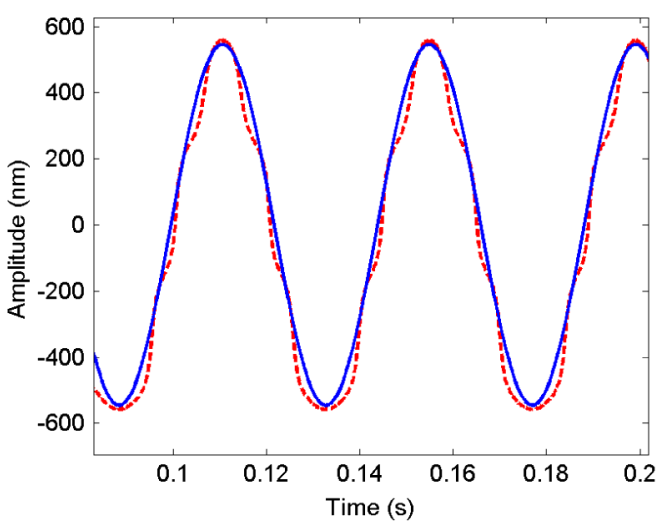

(b)

Fig. 9. (Color online) (a) I\&Q curve when the phase profile is different from a perfect sawtooth and the corresponding displacement deviations. In (b), the solid curve represents the original displacement signal and the dashed curve is the demodulated displacement when the fall time of the phase sawtooth is not small enough. In this case, the ratio between the fall time and the period in the phase sawtooth is $30 \%$. 


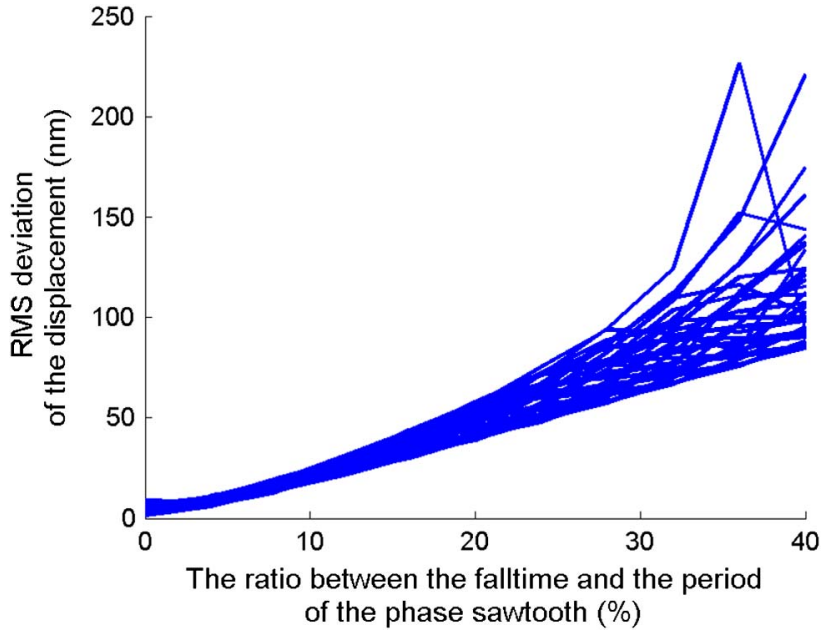

Fig. 10. (Color online) Simulated RMS deviations of the demodulated displacements versus the ratio of the fall time in the imperfect-phase sawtooth in the reference signal. The 36 solid curves represent 36 evenly spaced phases of the reference signal from 0 to $2 \pi$.

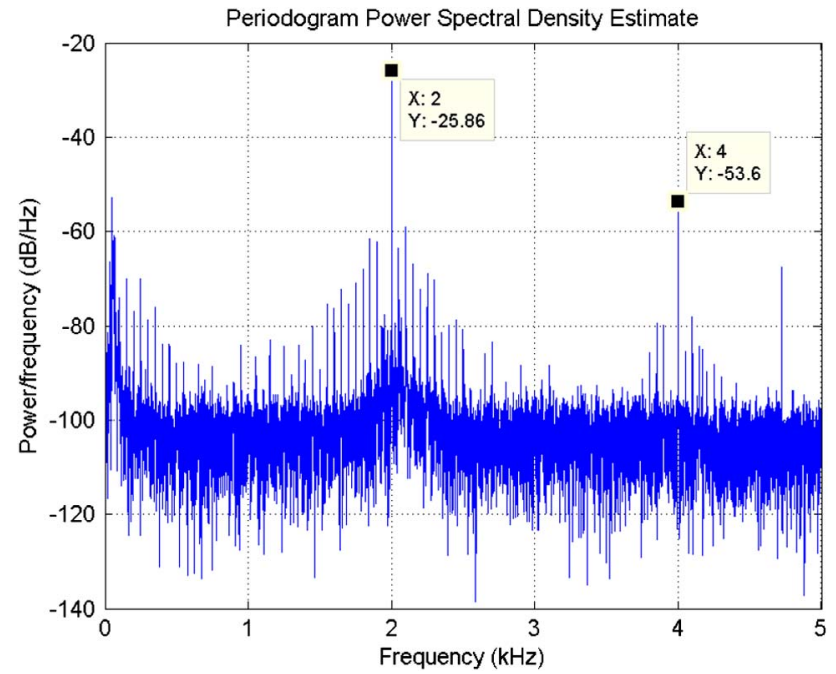

Fig. 11. (Color online) Power spectral density of the frequencyshifted signal.
The I\&Q traces for most of the measurement results are nice circles, which means that a proper serrodyne signal was generated. According to the measurement results, the power ratios between the recaptured useful reflection and the reference light are between -8 and $-14 \mathrm{~dB}$. The loss of the reflected light is mainly caused by the coupling loss in the grating coupler and imperfect alignment of the optical system. By estimating the modulation depth of the photocurrent while the vibrating surface is moved away and no useful reflection is sent back to the LDV, the ratio between the spurious reflection and the reference light can be obtained. This ratio turns out to be a stable value in time, which is about $-23 \mathrm{~dB}$. The major part of the spurious reflection probably comes from the $2 \times 2$ MMI. Because the stability of this ratio, the compensation method, which shifts the I\&Q trace center back to the origin, can thus work well. This compensation method will face problems if the useful reflection is too weak, in which case a small fluctuation in the spurious reflection can have a strong influence on the LDV output. According to the measured results, the compensation method can work well even when the power ratio between the recaptured useful light and the reference light reaches $-30 \mathrm{~dB}$, which means that the mirror can be replaced by a white paper. The grating coupler is good enough to be used for such a coupling efficiency. According to Eq. $\underline{3}$, the best suppression of -1st-order harmonic relative to the fundamental harmonic can be derived from the eccentricity of the I\&Q ellipse, and it is around $-42 \mathrm{~dB}$.

The signal-to-noise ratio (SNR) due to the shot noise can be calculated using

$$
\mathrm{SNR}=\frac{R P_{m} P_{r}}{q_{0} B\left(P_{r}+P_{m}\right)},
$$

where $P_{m}$ and $P_{r}$ are the power values of the measurement and reference signals, respectively, $R \approx$ $1 A / W$ is the responsivity of the detector, $q_{0}=$ $1.6 \times 10^{-19} C$ is the elementary charge, and $B$ is the bandwidth. According to this equation, the shotnoise-limited SNR value of the experimental results

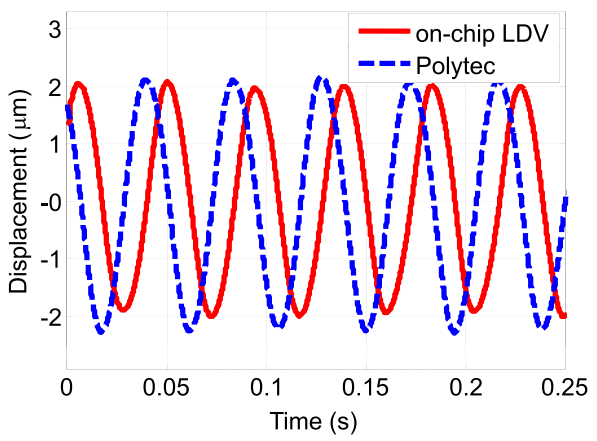

(a)

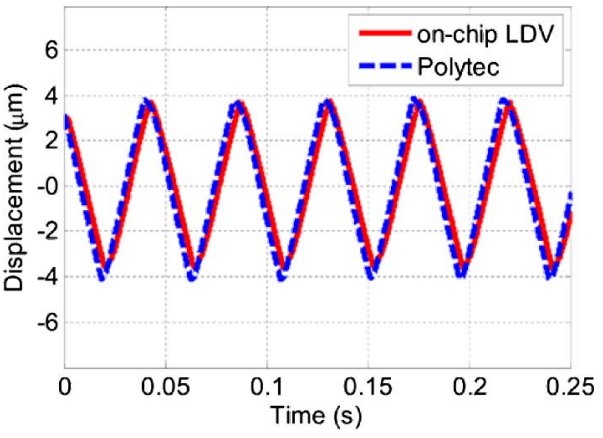

(b)

Fig. 12. (Color online) Demodulated displacements for on-chip LDV: the solid red curve stands for results measured with on-chip LDV, and the blue dashed curves are for results from Polytec LDV. Results in (a) are for the piezo vibration driven by a $50 V_{\mathrm{pp}}$ signal, while those in (b) are for the vibration driven by $100 V_{\mathrm{pp}}$. 


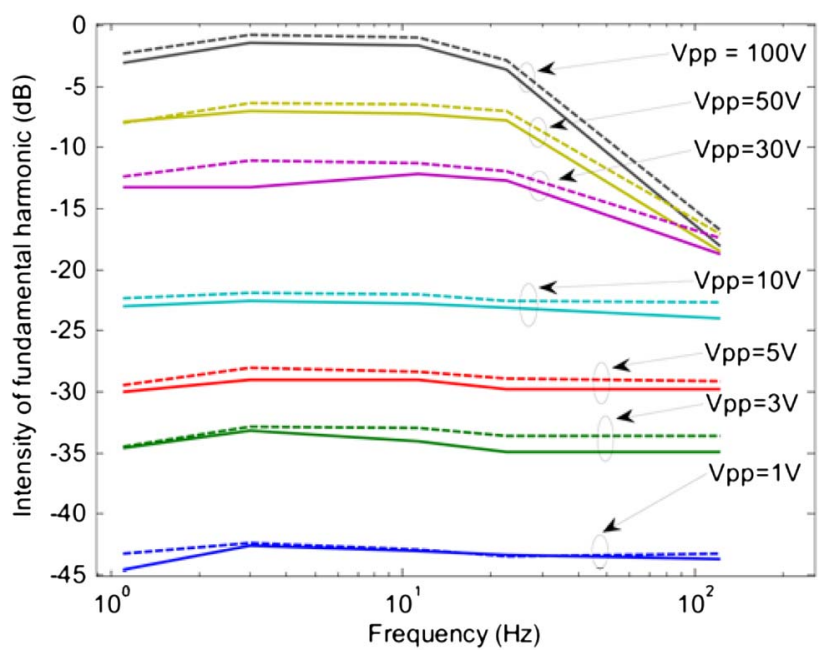

Fig. 13. (Color online) Response of the piezoelectric stack measured both by on-chip LDV (solid lines) and Polytec LDV (dashed lines). The peak-to-peak voltages of the piezo driver are chosen as $1,3,5,10,30,50$, and $100 \mathrm{~V}$.

is larger than $130 \mathrm{~dB}$. However, the quantization noise in the analog-to-digital converter (ADC) is around $-85 \mathrm{~dB}$ less than the signal, which is larger than the shot noise. This can be confirmed in the measurement result shown in Fig. 11. The power spectral density of the demodulated signal corresponding to Fig. 12(a) is plotted in Fig. 14. It can be found that the SNR is only $50 \mathrm{~dB}$ in this measurement, and this is because the environmental vibrations also have an influence on the mirror. The power spectral density of the background vibration is measured and is shown in Fig. 15. It can be found that the environmental vibration is mainly near $50 \mathrm{~Hz}$. In order to obtain the shot-noise limit, the quantization noise in the $\mathrm{ADC}$ has to be suppressed by increasing the ADC resolution, and a better compensation method should also be implemented.

Due to the bandwidth limitation of the OFS, the frequency and amplitude of the vibrations is limited according to Carson's bandwidth rule [14]: $f_{\text {ofs }}>2\left(f_{v}+f_{D}\right)$, where $f_{v}$ is the vibration frequency of the target, and $f_{D}$ is the Doppler frequency shift corresponding to the maximal velocity of the vibration. The maximal and minimal vibration velocities reported in this paper are around 400 and $0.8 \mu \mathrm{m} / \mathrm{s}$, respectively. The maximal $f_{D}$ is thus around $500 \mathrm{~Hz}$,

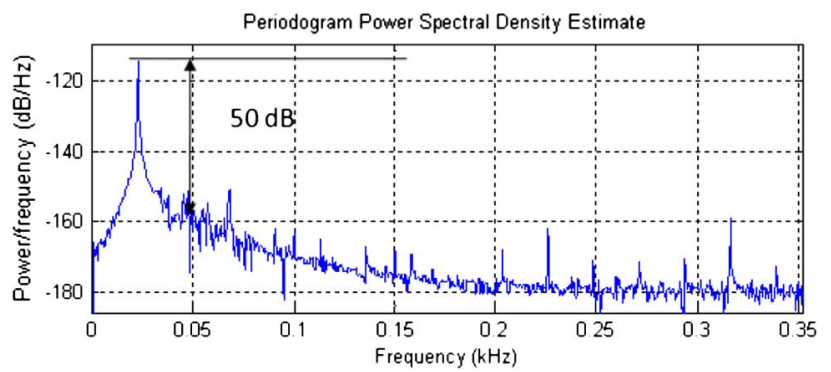

Fig. 14. (Color online) Power spectral density of the demodulated signal, when the piezo stack is driven by a $50 V_{\mathrm{pp}}$ signal at $22.6 \mathrm{~Hz}$.

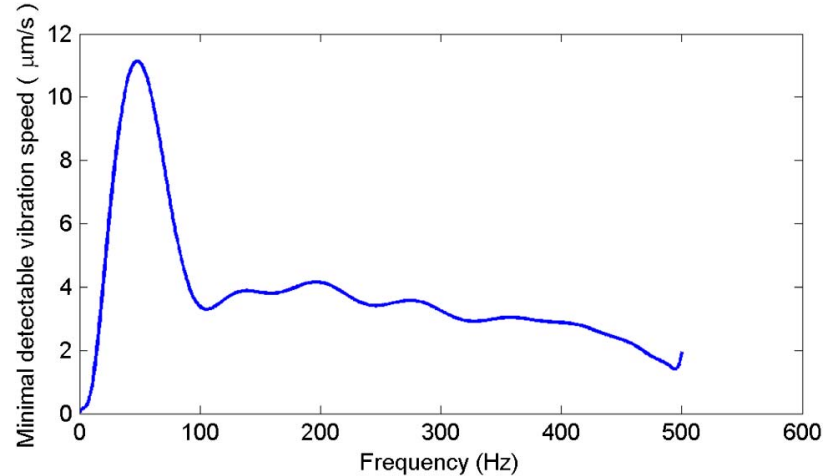

Fig. 15. (Color online) Power spectral density of environmental vibrations measured using LDV PIC with TO serrodyne frequency shift.

which means that it still fulfills Carson's rule if the vibration frequency is increased to $500 \mathrm{~Hz}$. This onchip LDV system can be useful in applications that only measure small vibrations at low frequencies. In order to measure vibrations with higher frequency or larger amplitudes, a faster OFS is needed. This might be realized by using a carrier injection/ depletion-based optical phase modulator, whose bandwidth could be larger than several gigahertz [9]. However, as mentioned, these phase modulators suffer from parasitic amplitude modulation, and appropriate measures are needed to mitigate the impact of amplitude modulation.

\section{Conclusion}

We have demonstrated an on-chip LDV with a TO phase-modulation-based serrodyne OFS. Vibrations of a piezo stack within the frequency range $[1.1 \mathrm{~Hz}, 123 \mathrm{~Hz}]$ and the velocity range between $[0.8 \mu \mathrm{m} / \mathrm{s}, 400 \mu \mathrm{m} / \mathrm{s}]$ are measured with the on-chip LDV. The measurement results are compared with a commercial LDV. It is shown that, with the help of a calibration factor, the on-chip LDV is able to retrieve the instantaneous displacements within this range. Theoretically, the measurable frequency range of the on-chip LDV can be extended to $500 \mathrm{~Hz}$ if the vibration is not larger than $400 \mu \mathrm{m} / \mathrm{s}$.

The power of the on-chip spurious reflection is about $23 \mathrm{~dB}$ less than that of the reference signal, and it will influence the LDV outputs if no compensation is made. Thanks to the stability of the on-chip interferometers, the spurious reflection is stable in time and the compensation can be realized. The compensation method works well if the power ratio between the useful reflection and reference light is kept larger than $-30 \mathrm{~dB}$, and this makes it possible to use a simple grating coupler on SOI for sending and receiving the measurement light.

The authors acknowledge the Ghent UniversityMethusalem project "Smart Photonic Chips" for financial support. The authors thank Xin Yin for useful discussions. 


\section{References}

1. H. Toda, M. Haruna, and H. Nishihara, "Optical integrated circuit for a fiber laser Doppler velocimeter," J. Lightwave Technol. 5, 901-905 (1987).

2. R. G. Brown, J. G. Burnett, J. Mansbridge, C. I. Moir, and B. S. Lowans, "Miniature, solid state photon correlation laser Doppler velocimetry," Appl. Opt. 29, 3291-3302 (1990).

3. A. Campo and J. Dirckx, "Dual-beam laser Doppler vibrometer for measurement of pulse wave velocity in elastic vessels," Proc. SPIE 8011, 80118Y (2011).

4. W. Bogaerts, R. Baets, P. Dumon, V. Wiaux, S. Beckx, D. Taillaert, B. Luyssaert, J. Van Campenhout, P. Bienstman, and D. Van Thourhout, "Nanophotonic waveguides in silicon-on-insulator fabricated with CMOS technology," J. Lightwave Technol. 23, 401-412 (2005).

5. A. T. Waz, P. R. Kaczmarek, and K. M. Abramski, "Laser-fibre vibrometry at $1550 \mathrm{~nm}$," Meas. Sci. Technol. 20, 105301 (2009).

6. T. O. H. Charrett, S. W. James, and R. P. Tatam, "Optical fibre laser velocimetry: a review," Meas. Sci. Technol. 23, 032001 (2012).

7. Y. Li, S. Meersman, and R. Baets, "Realization of fiber-based laser Doppler vibrometer with serrodyne frequency shifting," Appl. Opt. 50, 2809-2814 (2011).
8. Y. Li, S. Meersman, and R. Baets, "Optical frequency shifter on SOI using thermo-optic serrodyne modulation," Proceedings of 7th International Conference in Group IV Photonics (IEEE, 2010), p. P1.4.

9. W. Green, M. Rooks, and L. Sekaric, "Ultra-compact, low RF power, $10 \mathrm{~Gb} / \mathrm{s}$ silicon Mach-Zehnder modulator," Opt. Express 15, 17106-17113 (2007).

10. S. Stankovic, R. Jones, M. N. Sysak, J. M. Heck, G. Roelkens, and D. Van Thourhout, "Hybrid III-V/Si distributed-feedback laser based on adhesive bonding," IEEE Photon. Technol. Lett. 24, 2155-2158 (2012).

11. D. Vermeulen, Y. De Koninck, Y. Li, E. Lambert, W. Bogaerts, R. Baets, and G. Roelkens, "Reflectionless grating coupling for silicon-on-insulator integrated circuits," in Proceedings of 8th IEEE International Conference on Group IV Photonics (IEEE, 2011), pp. 74-76.

12. Y. Li, D. Vermeulen, Y. De Koninck, G. Yurtsever, G. Roelkens, and R. Baets, "Compact grating couplers on silicon-oninsulator (SOI) with reduced back reflection," Opt. Lett. 37, 4356-4358 (2012).

13. "OFV-534 Compact Sensor Head and OFV-5000 Modular Vibrometer Controller," www.polytec.com.

14. J. R. Carson, "Notes on the theory of modulation," Proc. IRE 10, 57-64 (1922) 